\title{
miR-205 regulates bone turnover in elderly female patients with type 2 diabetes mellitus through targeted inhibition of Runx2
}

\author{
GUANGFENG ZHANG ${ }^{1}$, HUAFENG LI ${ }^{2}$, WENJIE ZHAO ${ }^{2}$, MIN LI $^{2}$, \\ LINLIN TIAN ${ }^{2}$, WENWEN JU ${ }^{2}$ and XIAOBING LI $^{2}$ \\ Departments of ${ }^{1}$ MRI and ${ }^{2}$ Endocrinology (I), The Third Affiliated Hospital of Qiqihar Medical University, \\ Qiqihar, Heilongjiang 161099, P.R. China
}

Received June 14, 2019; Accepted January 30, 2020

DOI: $10.3892 /$ etm.2020.8867

\begin{abstract}
The present study aimed to explore the expression of microribonucleic acid (microRNA) (miR)-205 in bone tissues and serum of elderly female patients with type 2 diabetes mellitus (T2DM) complicated with osteoporosis (OP), and to investigate the effect of miR-205 on osteogenesis/adipogenesis of bone marrow mesenchymal stem cells (BMSCs) and its mechanism in elderly female mice with T2DM + OP. The bone tissues and serum of 24 female patients with T2DM + OP at the Third Affiliated Hospital of Qiqihar Medical University were collected as the research group, while those of 24 healthy people were collected as the control group. The expression level of miR-205 was detected in both groups via reverse transcription-polymerase chain reaction (RT-PCR). Then the elderly female mouse model of T2DM + OP was established as a model group, while normal mice of the same age were used as the control group. The mice in the model and control groups were transfected with $\mathrm{miR}-205 \mathrm{mimic}$, negative control (NC)-mimic, miR-205-inhibitor and NC-inhibitor. Alizarin red S (ARS) staining and RT-PCR were conducted after osteogenic induction for 21 days, and oil red $\mathrm{O}(\mathrm{ORO})$ staining and RT-PCR were performed after adipogenic induction for 24 days. The overexpression of miR-205 inhibited osteogenic differentiation and promoted adipogenic differentiation of BMSCs in elderly female mice with T2DM + OP, while knockdown of miR-205 promoted osteogenic differentiation and inhibited adipogenic differentiation of BMSCs in elderly female mice with T2DM + OP. In addition, miR-205 was able to directly suppress the expression of its target gene RUNX family transcription factor 2 (Runx2). The expression level of miR-205 was obviously increased in female patients with T2DM + OP and the elderly female mouse model of
\end{abstract}

Correspondence to: Dr Huafeng Li, Department of Endocrinology (I), The Third Affiliated Hospital of Qiqihar Medical University, 27 Taishun, Qiqihar, Heilongjiang 161099, P.R. China

E-mail: lihuafeng510@126.com

Key words: miRNA, type 2 diabetes mellitus, osteoporosis, osteogenesis, adipogenesis, bone turnover
T2DM + OP. In addition, miR-205 was able to regulate the osteogenic/adipogenic differentiation of BMSCs, and miR-205/Runx2 may be a new method and target for the treatment of female patients with $\mathrm{T} 2 \mathrm{DM}+\mathrm{OP}$.

\section{Introduction}

The ovarian function and estrogen level continuously decline in women relative to age, ultimately leading to osteoporosis (OP) (1). OP is one of the common complications of patients with type 2 diabetes mellitus (T2DM), causing great morbidity and seriously affecting the quality of life of patients (2-4). Currently, the main therapeutic methods for elderly patients with T2DM and OP include calcium and vitamin D supplements, physical exercise, specified diet and high quality nursing, yet the occurrence and progression of OP in elderly patients with T2DM cannot be effectively controlled $(5,6)$. Therefore, it is of great clinical significance to explore new therapeutic methods and drugs for patients with T2DM and OP.

Microribonucleic acids (microRNAs or miRs) are a type of single-stranded, non-coding and small-molecule RNAs approximately 18-25 nucleotides in length, which are involved in the occurrence and progression of various diseases $(7,8)$. It has been reported that some miRs can participate in bone turnover, bone metabolism and bone development, thereby regulating the occurrence and progression of OP (9-11). However, whether there is a certain association between miRs and T2DM + OP has not yet been reported.

In previous experiments, several miRNAs related to OP have been screened, and it was found that miR-205 had a certain association with T2DM + OP in elderly patients (9-11). In the present study, therefore, the expression of miR-205 in bone tissues and serum of elderly female patients with T2DM + OP was explored, and the effect of miR-205 on osteogenesis/adipogenesis of bone marrow mesenchymal stem cells (BMSCs) and its specific mechanism in elderly female mice with T2DM + OP were investigated, so as to provide new insight for the clinical treatment of elderly female patients with T2DM + OP.

\section{Materials and methods}

Patients and data. A total of 24 female patients with T2DM+OP treated at The Third Affiliated Hospital of Qiqihar Medical 
University (Qiqihar, Heilongjiang, China) from October 2016 to June 2017 were selected. The patients were aged from 60 to 80 years with an average age of $72.42 \pm 13.41$ years. All patients met the clinical diagnostic criteria for T2DM and the WHO diagnostic criteria for OP. WHO diagnostic criteria for T2DM included: Plasma glucose $\geq 11.1 \mathrm{mmol} / \mathrm{l}$, fasting plasma glucose (FPG) $\geq 7.0 \mathrm{mmol} / \mathrm{l}$, or oral glucose tolerance test 2 -h postprandial blood glucose (OGTT2hPG) $\geq 11.1 \mathrm{mmol} / 1$ at any time. WHO diagnostic criteria for OP included: A bone mineral density (BMD) measured by dual-energy X-ray absorptiometry to be 2.5 standard deviations below that in the local region and of the same gender and ethnic group ( $T$ value $\leq-2.5$ ). According to studies (12-17), when studying orthopedic-related diseases, the selected controls are usually patients admitted to the hospital for trauma. In this study, trauma that required emergency surgery due to multiple trauma to the bone was defined as severe trauma. At the same time, 24 patients with normal blood glucose and BMD who were treated at this hospital due to severe trauma were selected as the control group. During surgery of the elderly female patients with T2DM + OP and patients in the control group, the bone tissues were collected from the femur or tibia and immediately stored in liquid nitrogen. In addition, the fasting venous blood was collected in both groups in the morning, and the serum was separated and stored in a refrigerator at $-80^{\circ} \mathrm{C}$.

Exclusion criteria included patients with T1DM, special type of diabetes or autoimmune diseases, patients who presented with severe hematological diseases, those who received estrogen, calcitonin, vitamin $\mathrm{K}$ and other drugs that may affect bone metabolism for a long period prior to the study, those complicated with severe kidney disease, tumors or gout, patients who presented with other congenital OP, those who had poor mental or consciousness state or failed to cooperate in the study.

Establishment of an elderly female mouse model of $T 2 D M+O P$. All mice were maintained at a temperature between 20 and $26^{\circ} \mathrm{C}$, with a humidity of 40 to $60 \%$, and a 12-h light/12-h dark cycle, and free access to food and water. Animal health and behavior were monitored each morning and evening.

A total of $54 \mathrm{C} 57 \mathrm{BL} / 6 \mathrm{~J}$ female mice were obtained from Beijing Vital River Laboratory Animal Technology Co., Ltd. for this study, and $24 \mathrm{C} 57 \mathrm{BL} / 6 \mathrm{~J}$ female mice, 8 weeks of age, were used as the controls. Bone tissue and serum samples were collected from the mice. Thirty C57BL/6J female mice, age 16 months were used to construct the models. One week after acclimatization, a mouse model of osteoporosis was constructed. All mice were anesthetized with $50 \mathrm{mg} / \mathrm{kg}$ of $1 \%$ sodium pentobarbital. After anesthesia, under sterile conditions, ophthalmic scissors, ophthalmic tweezers, hemostatic forceps and other instruments were used to remove the bilateral ovaries of the mice. After suture, the mice were rested on a clean table, until they could move freely. Bilateral ovaries of the mice were removed. After 4 weeks of breeding, if the mice exhibited phenotypes such as reduced bone density, increased bone separation, and decreased number of trabeculae, the osteoporosis mouse model was deemed as successfully constructed. At the same time, the mice were fed with high-fat and high-glucose diets for 6 weeks, and
$2 \%$ streptozotocin (STZ) solution ( $\mathrm{pH} 4.4$ ) was intraperitoneally (i.p.) injected (35 mg/kg/time) after fasting for $12 \mathrm{~h}$, so as to establish the T2DM model. A FPG level of $>11.1 \mathrm{mmol} / 1$ within 2 weeks after STZ injection indicated T2DM. Finally, 24 mice met the criteria, and the elderly female mouse model of T2DM + OP was achieved after 8 weeks. The bone tissues and serum samples were collected in both groups for subsequent experiment. Once it was clear that the 6 mice did not meet the requirement of subsequent experiment, the experiments on the 6 mice were stopped immediately, and euthanasia was performed. In 'Guide for the Care and Use of Laboratory Animals' of the National Institutes of Health, endpoints for experiments are defined. It is highlighted that euthanasia is performed to reduce animal pain and suffering before death. Dying is the state before the death of the animal, which can replace death as the experimental endpoint. The criteria for the dying end point include the following: i) weight loss; ii) body temperature reduction; iii) obvious inactivity or remain motionless; iv) arched back posture; v) hair stains; vi) tumor ulceration and bleeding; vii) dyspnea. In this experiment, we found that 6 mice did not meet the requirement of subsequent experiment, and because of turbid hair and arched back, they were euthanized. The other 6 mice were sacrificed by injection of $200 \mathrm{mg} / \mathrm{kg}$ sodium pentobarbital.

To summarize, in the present study, 54 female C57BL/6J mice were utilized, and 248 -week-old control mice were also sacrificed by cervical dislocation after anesthesia. Thirty 16-month-old mice were used to construct the model of type 2 diabetes with osteoporosis; among them, 6 mice did not meet the standard and were euthanized, and 24 mice were sacrificed by cervical dislocation after anesthesia.

Total RNA extraction and reverse transcription-polymerase chain reaction ( $R T-P C R)$. Total RNA was extracted from bone tissues using TRIzol reagent (Invitrogen; Thermo Fisher Scientific, Inc.), and the miRNA was extracted from serum using a serum miRNA extraction kit (Foregene). The RNA purity and concentration were detected using a Nanodrop spectrophotometer (Thermo Fisher Scientific, Inc.). Then RNA was synthesized into cDNA using the RevertAid ${ }^{\mathrm{TM}}$ First Strand cDNA Synthesis kit (Fermentas) and amplified using the SYBR Green Master Mix (Roche) on the ABI7500 PCR instrument (Applied Biosystems; Thermo Fisher Scientific, Inc.) to detect the expression of the target gene. All primer sequences used in this experiment are shown in Table I.

Separation and culture of mouse BMSCs. After the successful establishment of the elderly female mouse model of T2DM + OP, mouse bone marrow mesenchymal stem cells (BMSCs) were extracted according to a previous study (18). First, the mice were anesthetized by an i.p. injection of sodium barbiturate (Sigma-Aldrich; Merck KGaA), and then the mice were sacrificed by cervical dislocation. The bilateral femur and tibia were isolated under sterile conditions; muscle tissue was removed, and the femur and tibia were exposed. Then BMSCs were cultured in high-glucose DMEM (Corning) containing 10\% fetal bovine serum (FBS) (Cyagen), $100 \mathrm{U} / \mathrm{ml}$ penicillin (Beyotime) and $100 \mu \mathrm{g} / \mathrm{ml}$ streptomycin (Beyotime). The medullary cavity was rinsed repeatedly, the cells were extracted out of the marrow cavity, centrifuged with 
Table I. Primer sequences.

Gene

Murine miR-205

Human miR-205

Murine U6

Human U6

Murine $A L P$

Murine $O C N$

Murine PPAR $\gamma$

Murine FABP4

Murine Runx2

Murine $\beta$-actin
Forward (5'-3')

Reverse (5'-3')

$U 6$, RNA, U6 small nuclear 1; $A L P$, alkaline phosphatase, biomineralization associated; $O C N$, osteocalcin [also known as bone $\gamma$-carboxyglutamate protein $(B G L A P)]$; PPAR $\gamma$, peroxisome proliferator-activated receptor- $\gamma ; F A B P 4$, fatty acid binding protein $4 ;$ Run $x 2$, runt-related transcription factor 2 .

a high-speed centrifuge (Thermo Fisher Scientific, Inc.) for $5 \mathrm{~min}$ at $200 \mathrm{x}$ g, and the cell pellet was placed in cell bottles. Then the cell pellet was placed in an incubator (Thermo Fisher Scientific, Inc.) with $5 \% \mathrm{CO}_{2}$ at $37^{\circ} \mathrm{C}$. BMSCs were purified by differential adherence method; culture medium was replaced every three days, and non-adherent cells were removed; the splitting ratio was $1: 3$. The above steps were repeated and passed to the third generation for subsequent experiments. The study was approved by the Animal Ethics Care Committee of Qiqihar Medical University (QMU-AECC-2019-51).

Cell transfection. The third-generation BMSCs in the logarithmic growth phase were inoculated into a 24-well plate $\left(2 \times 10^{4} / \mathrm{cm}^{2}\right)$, and transiently transfected using the transfection reagent X-treme (Vazyme). After transfection with the miR-205 mimic (final concentration: $50 \mathrm{nM}$ ) and miR-205 inhibitor (final concentration: $100 \mathrm{nM}$ ) for $24 \mathrm{~h}$, subsequent experiments were performed. The sequences were: miR-205 mimic, 5'-UCCUUC AUUCCACCGGAGUCUG-3' and NC-mimic, 5'-UUCUCC GAACGUGUCACGUTT-3'; miR-205 inhibitor, 5'-CAGACU CCGGUGGAAUGAAGGA-3' and NC-inhibitor, 5'-CAGUAC UUUUGUGUAGUACAA-3'.

$C C K-8$ assay. BMSCs in the third-generation logarithmic growth phase were selected and seeded in 96-well plates at a density of $1 \times 10^{4}$ cells $/ \mathrm{cm}^{2}$. The cells were transfected with miR-205. After 24, 48 and $72 \mathrm{~h}, 10 \mu \mathrm{l}$ of CCK-8 solution and $100 \mu \mathrm{l}$ of serum-free medium were added to each well. The cells were cultured for $2 \mathrm{~h}$ in an incubator, and the absorbance value (OD value) of each well was measured at $450 \mathrm{~nm}$ using a microplate reader (Tecan).

Osteogenic and adipogenic induction of BMSCs. The third-generation BMSCs in the logarithmic growth phase were inoculated into a 24 -well plate $\left(2 \times 10^{4} / \mathrm{cm}^{2}\right)$ and transfected with miR-205. Then osteogenic induction medium containing $10 \% \mathrm{FBS}, 10 \mathrm{mmol} / \mathrm{l}$ sodium 3-phosphoglycerate, $10 \mathrm{~mol} / \mathrm{l}$ dexamethasone and $50 \mu \mathrm{g} / \mathrm{ml}$ ascorbic acid was added for osteogenic induction for 21 days, and the original medium was replaced with fresh medium once every 3 days. After that, alizarin red S (ARS) staining was performed. First, the induction medium was discarded, and the cells were washed with PBS repeatedly, fixed with $4 \%$ paraformaldehyde for $30 \mathrm{~min}$, stained with ARS dye (Cyagen) for $30 \mathrm{~min}$ and washed again with PBS, followed by observation under an inverted microscope (magnification, $\mathrm{x} 10$ ).

In addition, the third-generation BMSCs in the logarithmic growth phase were inoculated into a 24 -well plate and transfected with miR-205. Then adipogenic induction medium containing $1 \mu \mathrm{M}$ of dexamethasone, $200 \mu \mathrm{M}$ of indometacin, $0.5 \mathrm{mM}$ of IBMX and $10 \mu \mathrm{M}$ of insulin for adipogenic induction for 24 days, and the original medium was replaced with the fresh medium once every 3 days. After that, oil red O (ORO) staining was performed. First, the induction medium was discarded, and the cells were washed with PBS 3 times, fixed with $4 \%$ paraformaldehyde at room temperature for $30 \mathrm{~min}$, stained with ORO dye (Cyagen) for $30 \mathrm{~min}$ and washed again with PBS, followed by observation under the inverted microscope (magnification, $\mathrm{x} 10$ ).

Target prediction. The target genes of miR-205 were predicted and analyzed using the online websites TargetScan (http://www. targetscan.org/vert_72/) and miRanda (http://www.microrna. org/src/jmiranda/).

Western blotting. The total protein was lysed using protein lysis buffer (Beyotime) and centrifuged at 2,500 x g and $4^{\circ} \mathrm{C}$ for $5 \mathrm{~min}$. The supernatant was transferred into another EP tube and cryopreserved in a refrigerator at $-80^{\circ} \mathrm{C}$. The protein concentration was measured using the Bradford protein quantification kit, and loading buffer was added to the samples and boiled. After $12.5 \%$ SDS-PAGE, the protein samples were transferred onto a PVDF membrane, sealed with skim milk for $2 \mathrm{~h}$, and incubated with the primary antibodies, Runx2 (dilution 1:1,000, cat. no. AF2593; Beyotime) or GAPDH (dilution 1:1,000, cat. no. AF1186; Beyotime) on a shaker at $4^{\circ} \mathrm{C}$ overnight. On the next day, the protein samples were incubated with the anti-rabbit secondary antibody (dilution, 1:500; cat. no. A0279; Beyotime) at room temperature. After $1 \mathrm{~h}$, the fluorescence signals were collected using the luminescence 
detector and analyzed by Image-Pro Plus 6 software (Media Cybernetics).

Luciferase reporter assay. The miR-205 precursor sequences and the 3'-untranslated region (3'UTR) of the mouse Runx2 gene were cloned into the psi-CHECK2 vector through PCR, enzyme digestion, ligation and transformation, and $0.5 \mu \mathrm{g}$ of 3'UTR and $1 \mu \mathrm{g}$ of miR were co-transfected into BMSCs. After $48 \mathrm{~h}$, the cells were collected and lysed. According to the instructions of the dual-luciferase reporter gene kit (Promega), the firefly luciferase activity and Renilla luciferase activity were detected for luciferase reporter assay.

Statistical methods. All data are expressed as mean \pm standard error of measurement, and a t-test was performed for the comparison of sample means. GraphPad 7.0 software (GraphPad Software, Inc.) was used for the statistical processing of all data, and the t-test for statistical analysis. ${ }^{*} \mathrm{P}<0.05,{ }^{* *} \mathrm{P}<0.01$ and ${ }^{* * *} \mathrm{P}<0.001$ were indicative of statistically significant differences as shown in the figures and defined in the figure legends.

\section{Results}

Expression of miR-205 is increased in elderly female patients with $T 2 D M+O P$. Compared with the control group, the expression level of miR-205 was significantly increased in the bone tissues and serum of elderly female patients with $\mathrm{T} 2 \mathrm{DM}+\mathrm{OP}(\mathrm{P}=0.0098$ and $\mathrm{P}=0.001)($ Fig. $1 \mathrm{~A}$ and $\mathrm{B})$.

Expression of miR-205 is increased in the elderly female mouse model of T2DM +OP. The expression level of miR-205 was higher in the bone tissues, serum and BMSCs of the elderly female mice with T2DM + OP than this level in the control group (Fig. 2A-C).

Effect of miR-205 on BMSC viability in the elderly female mice with $T 2 D M+O P$. To investigate the effect of miR-205 overexpression on the biological function of BMSCs in elderly female type 2 diabetic mice with OP, the cells were transfected with the miR-205 mimic and the transfection was deemed successful (Fig. 3A). CCK-8 assay was performed to explore the effect of miR-205 on the cell viability of BMSCs extracted from the elderly female mice with T2DM + OP. The results showed that overexpression of miR-205 significantly inhibited the viability of BMSCs in the elderly female mice with T2DM + OP when compared to the negative control (NC) group (Fig. 3B). To investigate the effect of miR-205 knockdown on the biological function of BMSCs in elderly female type 2 diabetic mice with OP, the cells were transfected with the miR-205 inhibitor and the transfection was deemed successful (Fig. 3A). Knockdown of miR-205 significantly increased the viability of BMSCs in elderly female mice with $\mathrm{T} 2 \mathrm{DM}+\mathrm{OP}$ when compared to the NC group (Fig. 3C).

Effects of overexpression of miR-205 on osteogenic/adipogenic differentiation of BMSCs in elderly female mice with $T 2 D M+O P$. The results of ARS staining showed that overexpression of miR-205 obviously inhibited osteogenic differentiation of BMSCs in the elderly female mice with
A

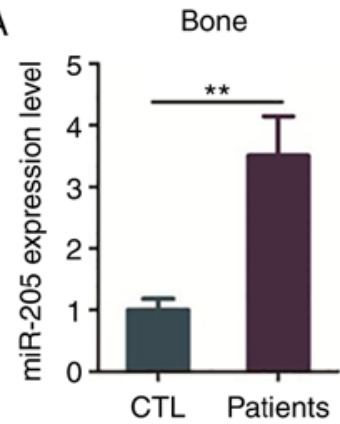

B

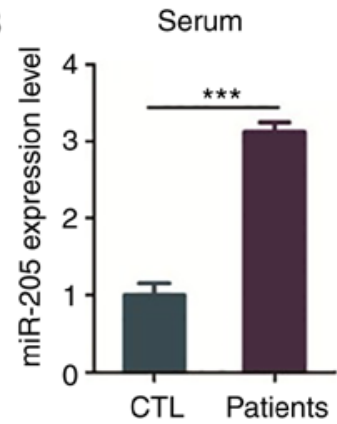

Figure 1. Expression level of miR-205 in the elderly female patients with T2DM + OP. Expression level of miR-205 in (A) bone tissues and (B) serum. ${ }^{* *} \mathrm{P}<0.01$ and ${ }^{* * * *} \mathrm{P}<0.001$, compared to the control (CTL). T2DM, type 2 diabetes mellitus; OP, osteoporosis.

T2DM + OP (Fig. 4A). In addition, the results of RT-PCR revealed that overexpression of miR-205 obviously reduced the expression of the osteogenesis-related genes, alkaline phosphatase, biomineralization associated $(A L P)$ and osteocalcin $(O C N)$ in BMSCs of elderly female mice with T2DM + OP (Fig. 4B).

Moreover, BMSCs of elderly female mice with T2DM + OP were transfected with miR-205 mimic and negative control (NC)-mimic, and ORO staining and RT-PCR were carried out after adipogenic induction for 24 days. The results of ORO staining showed that overexpression of miR-205 obviously promoted adipogenic induction of BMSCs in elderly female mice with T2DM + OP (Fig. 4C). In addition, the results of RT-PCR revealed that overexpression of miR-205 obviously increased the expression of adipogenesis-related genes $P P A R \gamma$ and FABP4 in BMSCs of elderly female mice with $\mathrm{T} 2 \mathrm{DM}+\mathrm{OP}$ (Fig. 4D).

Effects of knockdown of miR-205 on osteogenic/adipogenic differentiation of BMSCs in elderly female mice with $T 2 D M+O P$. BMSCs of elderly female mice with T2DM + OP were transfected with miR-205 inhibitor and NC-inhibitor, and ARS staining and RT-PCR were performed after osteogenic induction for 21 days. The results of ARS staining showed that knockdown of miR-205 markedly promoted osteogenic differentiation of BMSCs in elderly female mice with $\mathrm{T} 2 \mathrm{DM}+\mathrm{OP}$ (Fig. 5A). In addition, the results of RT-PCR revealed that knockdown of miR-205 significantly upregulated the expression of osteogenesis-related genes $A L P$ and $O C N$ (Fig. 5B).

Moreover, BMSCs of elderly female mice with T2DM + OP were transfected with miR-205 inhibitor and NC-inhibitor, and ORO staining and RT-PCR were conducted after adipogenic induction for 24 days. The results of ORO staining showed that the knockdown of miR-205 markedly suppressed adipogenic induction of BMSCs in elderly female mice with T2DM + OP (Fig. 5C). In addition, the results of RT-PCR revealed that the knockdown of miR-205 significantly downregulated the expressions of adipogenesis-related genes PPAR $\gamma$ and FABP4 (Fig. 5D).

miR-205 inhibits Runx2 expression in a targeted manner. The target genes of miR-205 were predicted using online websites 

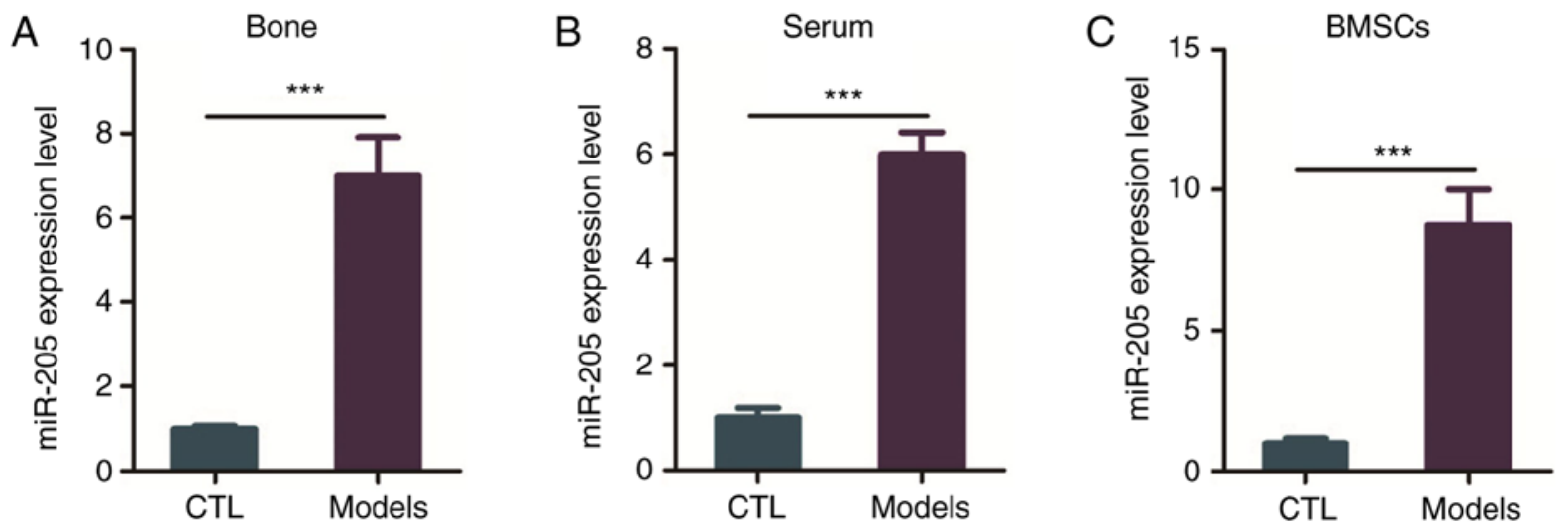

Figure 2. Expression level of miR-205 in the elderly female mouse model of T2DM + OP. Expression level of miR-205 in (A) bone tissues, (B) serum and (C) BMSCs. ${ }^{* * *} \mathrm{P}<0.001$, compared to the control (CTL). T2DM, type 2 diabetes mellitus; OP, osteoporosis; BMSCs, bone marrow mesenchymal stem cells.
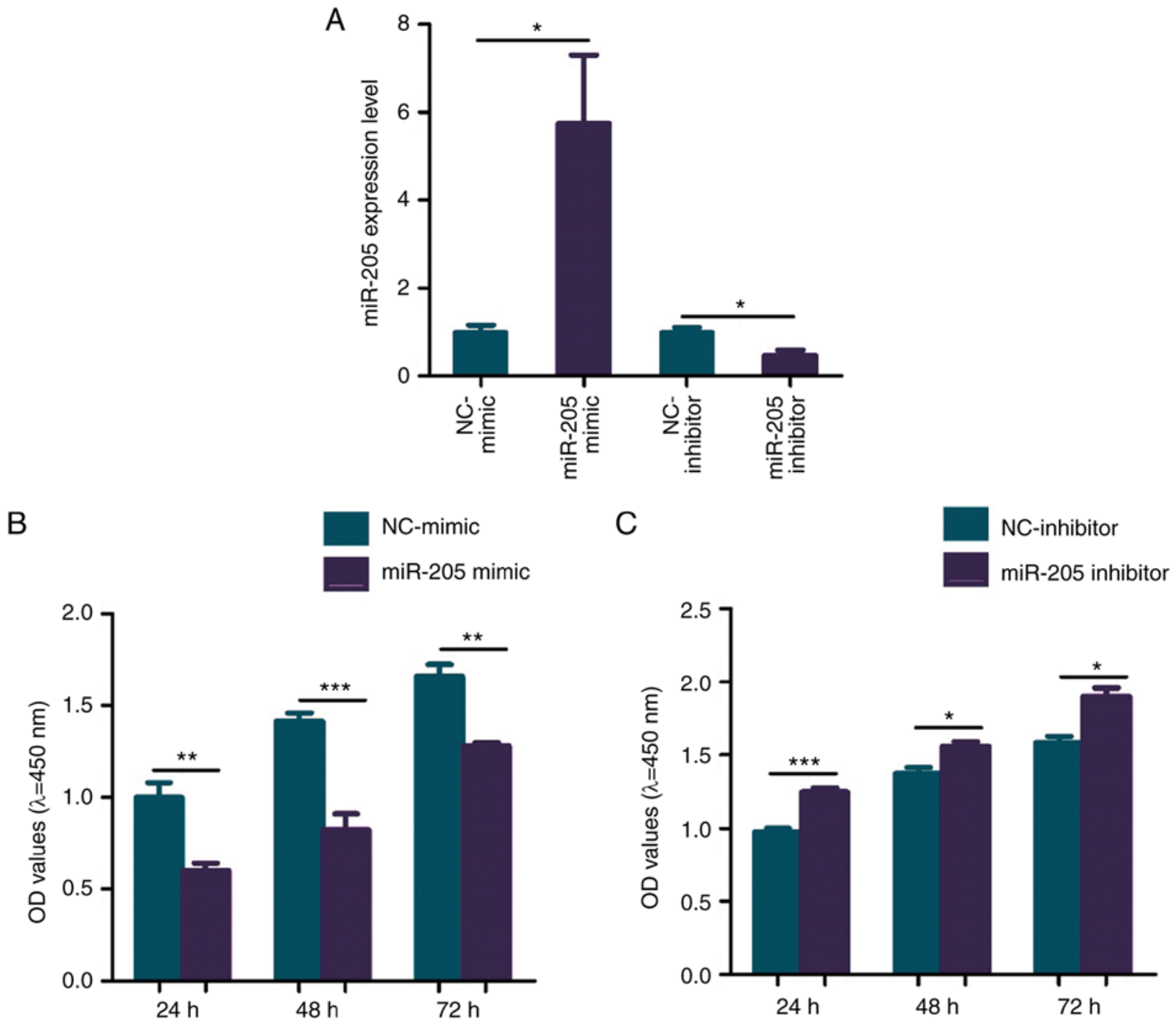

Figure 3. Effect of overexpression and knockdown of miR-205 on the viability of BMSCs in female mice with T2DM + OP. (A) Transfection efficiency of miR-205 mimic and inhibitor compared with the NC groups. (B and C) CCK-8 assay was used to detect the cell viability (OD value) of each group of BMSCs. ${ }^{*} \mathrm{P}<0.05,{ }^{* *} \mathrm{P}<0.01$ and ${ }^{* * *} \mathrm{P}<0.001$ compared to the relative NC group. T2DM, type 2 diabetes mellitus; OP, osteoporosis; BMSCs, bone marrow mesenchymal stem cells; OD, optical density; NC, negative control.

TargetScan and miRanda, and the binding sites for miR-205 and Runx2 are shown in Fig. 6A. Luciferase reporter assay confirmed that there was a regulatory relationship between
miR-205 and Runx2 (Fig. 6B). BMSCs of elderly female mice with $\mathrm{T} 2 \mathrm{DM}+\mathrm{OP}$ were transfected with miR-205 mimic, NC-mimic, miR-205 inhibitor and NC-inhibitor, after which 
A

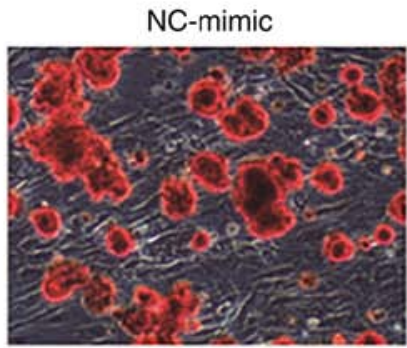

C

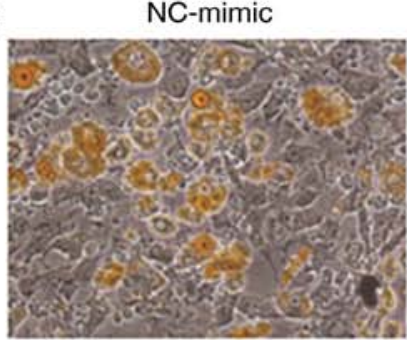

miR-205 mimic

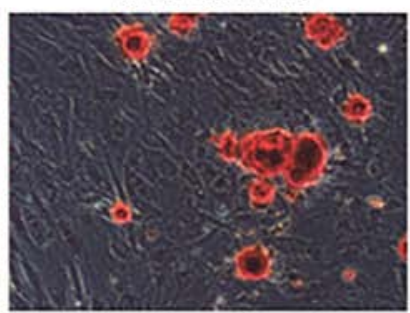

miR-205 mimic

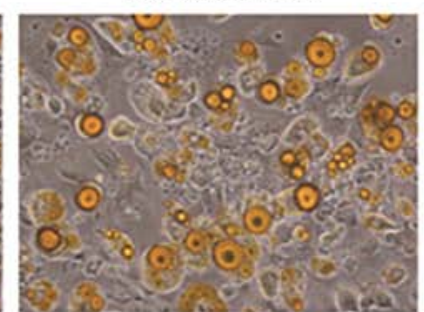

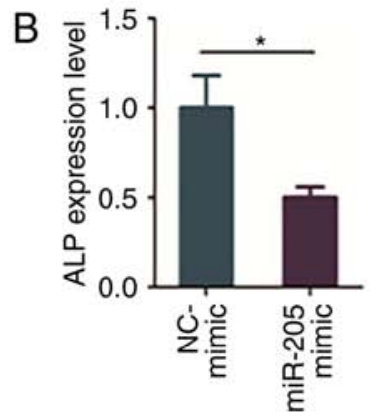

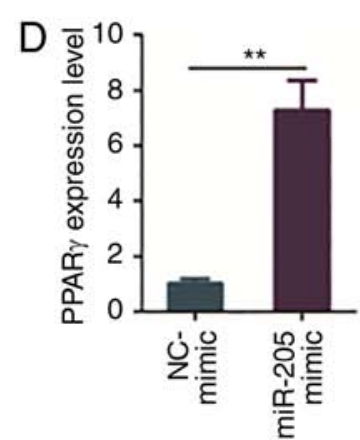

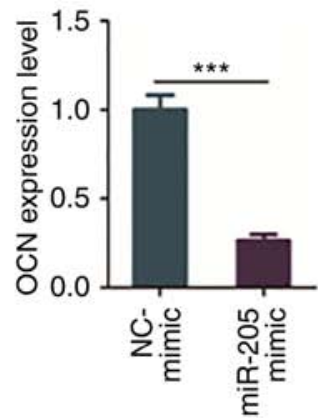

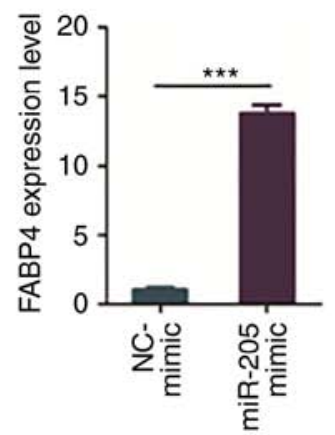

Figure 4. Overexpression of miR-205 inhibits osteogenic differentiation and promotes adipogenic differentiation of BMSCs in elderly female mice with T2DM + OP. (A) Osteogenic differentiation of BMSCs as detected via ARS staining. (B) Expression levels of osteogenesis genes, $A L P$ and $O C N$, as detected via RT-PCR. (C) Adipogenic differentiation as detected via ORO staining. (D) Expression levels of adipogenesis genes, $P P A R \gamma$ and $F A B P 4$, as detected via RT-PCR. ${ }^{*} \mathrm{P}<0.05,{ }^{* *} \mathrm{P}<0.01$ and ${ }^{* * *} \mathrm{P}<0.001$ compared to the NC-mimic group. BMSCs, bone marrow mesenchymal stem cells; T2DM, type 2 diabetes mellitus; OP, osteoporosis; ARS, alizarin red S; ORO, oil red O; ALP, alkaline phosphatase, biomineralization associated; $O C N$, osteocalcin; PPAR $\gamma$, peroxisome proliferator-activated receptor- $\gamma ; F A B P 4$, fatty acid binding protein 4.

A

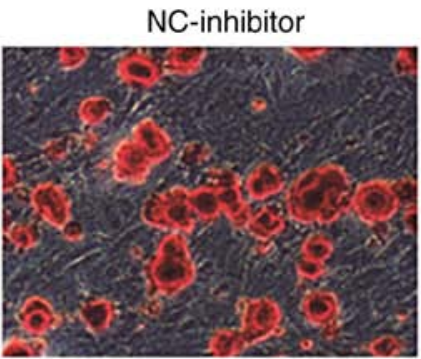

C

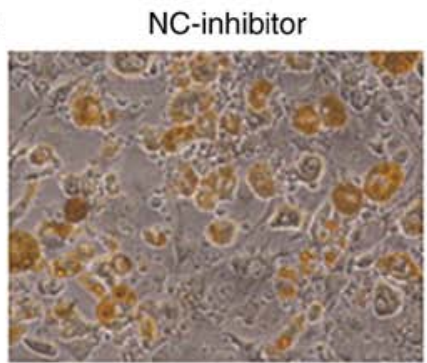

miR-205 inhibitor

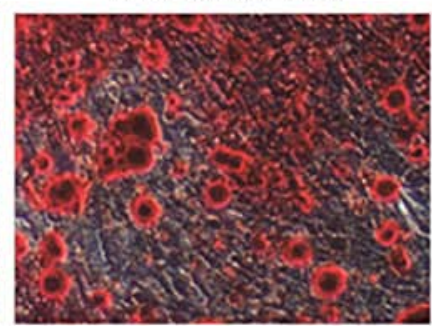

miR-205 inhibitor

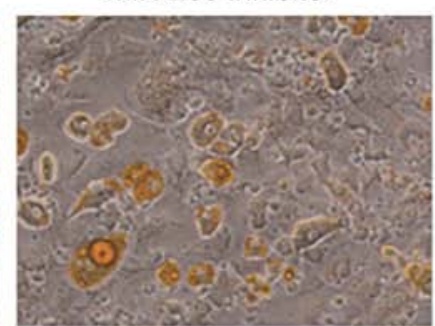

B
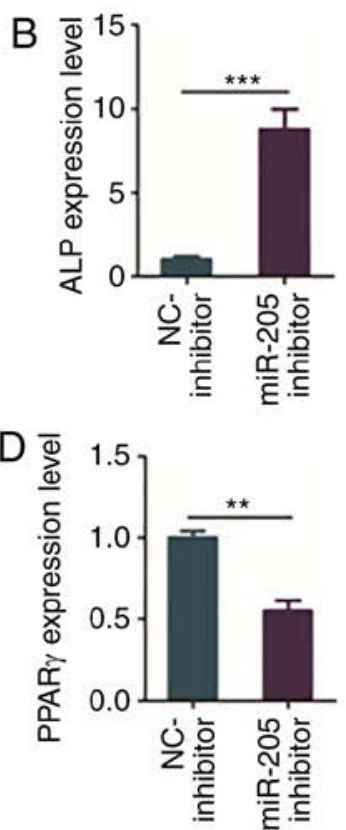
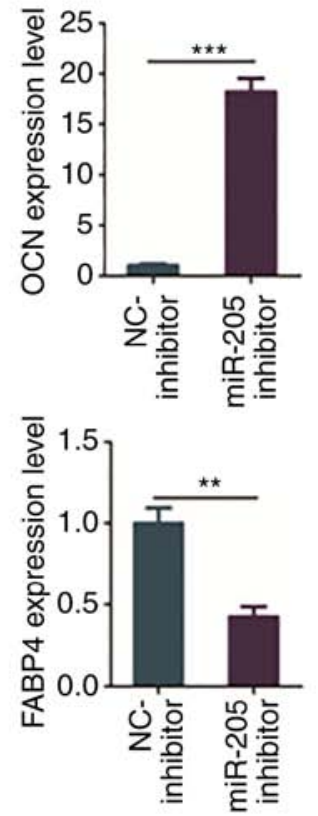

Figure 5. Knockdown of miR-205 promotes osteogenic differentiation and inhibits adipogenic differentiation of BMSCs in elderly female mice with T2DM + OP. (A) Osteogenic differentiation of BMSCs detected via ARS staining. (B) Expression levels of osteogenesis genes, $A L P$ and $O C N$, as detected via RT-PCR. (C) Adipogenic differentiation as detected via ORO staining. (D) Expression levels of adipogenesis genes, $P P A R \gamma$ and $F A B P 4$, as detected via RT-PCR. ${ }^{* *} \mathrm{P}<0.01$ and ${ }^{* * *} \mathrm{P}<0.001$ compared to the NC-inhibitor group. BMSCs, bone marrow mesenchymal stem cells; T2DM, type 2 diabetes mellitus; OP, osteoporosis; ARS, alizarin red S; ORO, oil red O; $A L P$, alkaline phosphatase, biomineralization associated; $O C N$, osteocalcin; PPAR $\gamma$, peroxisome proliferator-activated receptor- $\gamma ; F A B P 4$, fatty acid binding protein 4.

the mRNA and protein levels of Runx2 were determined. It was found that overexpression of miR-205 significantly suppressed the mRNA expression of Runx2, while knockdown of miR-205 significantly increased the mRNA expression of Runx2 (Fig. 6C). As shown in Fig. 6D, the results of western blotting were consistent with those of RT-PCR. Overexpression 

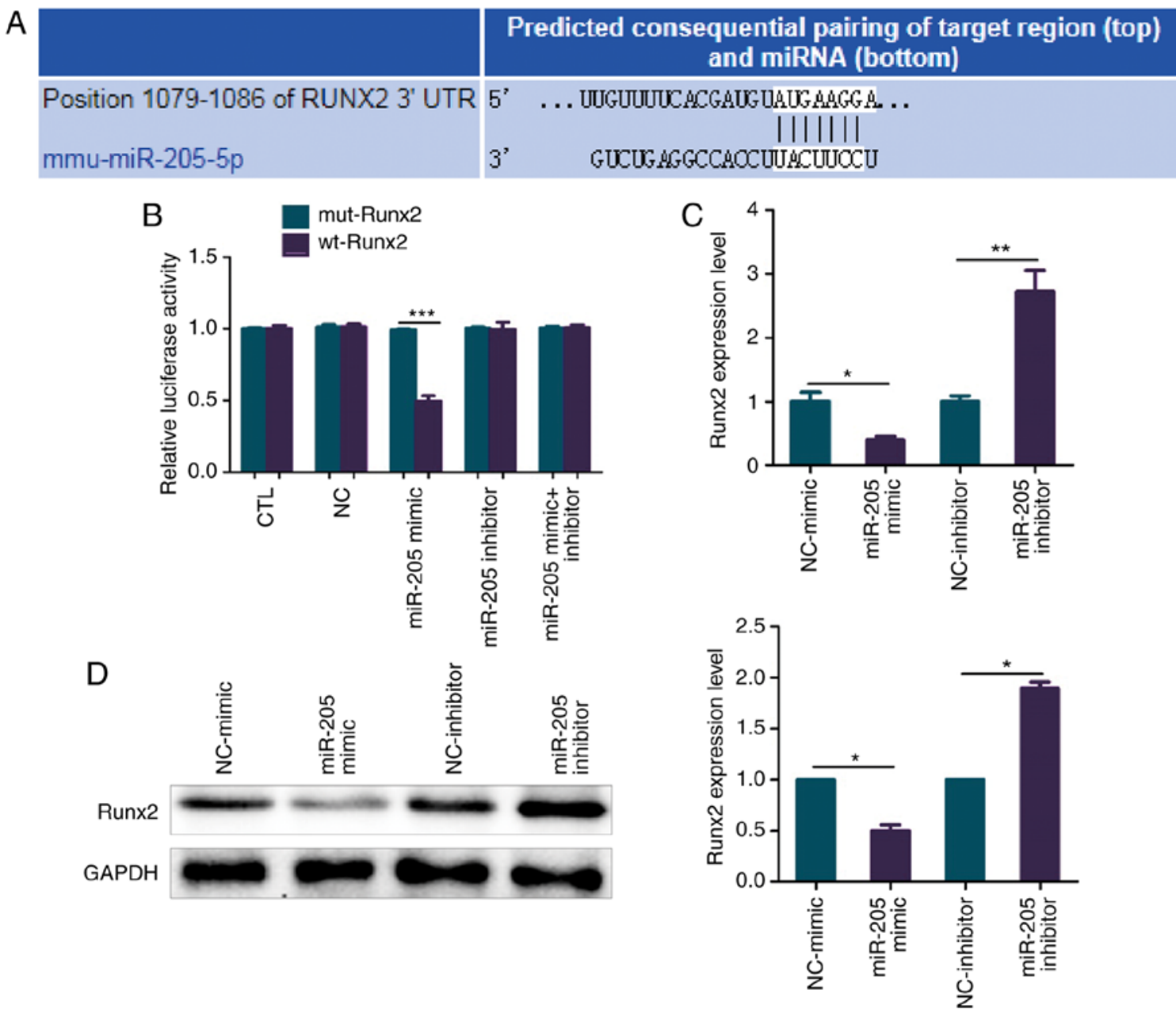

Figure 6. Runx2 is a direct target gene of miR-205. (A) Binding sites for Runx2 and miR-205. (B) Correlation between miR-205 and Runx2 as detected via luciferase reporter assay. (C) Runx 2 mRNA level as detected via RT-PCR. (D) Runx2 protein level as detected via western blotting. " $\mathrm{P}<0.05$, ${ }^{* *} \mathrm{P}<0.01$ and ${ }^{* * *} \mathrm{P}<0.001$. Runx2, RUNX family transcription factor 2 ; CTL, control; NC, negative control.

of miR-205 significantly suppressed the protein expression of Runx2, while knockdown of miR-205 significantly increased the protein expression of Runx2 (Fig. 6D).

\section{Discussion}

Osteoblasts are the most important cells in the bone microenvironment, accounting for approximately $95 \%$ of the total cell population in bone tissues, and they can determine the proportion of components in bone tissues, whose main source is bone marrow mesenchymal stem cells (BMSCs) $(19,20)$. Under certain conditions, BMSCs can differentiate into osteoblasts, adipocytes and chondrocytes, and there is a certain dynamic balance between osteogenic and adipogenic differentiation of BMSCs under normal conditions, which plays an important role during bone development (21-23).

Studies have found that miRNAs, as ubiquitous molecules that regulate gene expression, play important regulatory roles in bone development and bone formation (24). For example, Yang et al found that miR-21 was able to facilitate bone formation and bone regeneration through PTEN/PI3K/Akt/HIF-1 $\alpha$ (25). Therefore, whether there is an miRNA able to regulate osteogenic/adipogenic differentiation of BMSCs in elderly female patients with T2DM + OP was explored in the present study.
In previous studies, the expression levels of miRNAs (miR-205, miR-188, miR-23a, miR-26a and miR-214) related to bone development and bone formation were detected in elderly female patients with T2DM + OP (9-11), and the results showed that the expression of miR-205 was significantly upregulated in bone tissues and serum of elderly female patients with T2DM + OP, thus miR-205 was selected as the object of further study. It is reported that miR-205 can regulate the biological functions of a variety of cancer cells and participate in bone formation. However, the correlation between miR-205 and osteogenic/adipogenic differentiation of BMSCs in elderly female patients with T2DM + OP has been sparsely studied. Therefore, the expression of miR-205 in bone tissues, serum and BMSCs of elderly female mice with T2DM + OP and its effect on osteogenic/adipogenic differentiation of BMSCs was further investigated. It was found that the expression of miR-205 was obviously increased in elderly female patients with T2DM + OP and elderly female mice with $\mathrm{T} 2 \mathrm{DM}+\mathrm{OP}$, which provides an important target and marker for the early prediction and diagnosis of T2DM + OP in the elderly. Hu et al (26) found that the miR-205 expression obviously declined during osteogenic differentiation of normal BMSCs, consistent with our conjecture. In the present study, overexpression of miR-205 inhibited osteogenic differentiation and promoted adipogenic differentiation 
of BMSCs in elderly female mice with T2DM + OP, while knockdown of miR-205 promoted osteogenic differentiation and inhibited adipogenic differentiation of BMSCs in elderly female mice with T2DM + OP. Hu et al (26) found that miR-205 can weaken osteogenic differentiation and enhance adipogenic differentiation of normal BMSCs. Therefore, we conclude that miR-205 inhibits osteogenic differentiation and promotes adipogenic differentiation of BMSCs in normal mice and elderly female mice with T2DM + OP. In addition, the results of luciferase reporter assay, RT-PCR and western blotting confirmed that miR-205 could directly inhibit the expression of its target gene Runx2. Runx2 is an important regulator of osteogenic differentiation and is able to regulate the transcription of various osteogenesis-related genes $(27,28)$. Inhibiting Runx2 expression may affect osteogenic differentiation of BMSCs, thereby impacting the formation of osteoblasts and ultimately affecting bone development and bone formation (29). We will reorganize the phenotype of BMSCs in vitro in future research, and perform subcutaneous implantation experiments of BMSCs from nude mice with different expression levels of miR-205. We suspect that the subcutaneous implantation of BMSCs with overexpressing miR-205 can inhibit the formation of subcutaneous bone in nude mice, and the subcutaneous implantation of BMSCs with silenced miR-205 can promote the formation of subcutaneous bone in nude mice. Therefore, it was found in the present study that miR-205 is involved in the osteogenic/adipogenic differentiation of BMSCs in elderly female mice with $\mathrm{T} 2 \mathrm{DM}+\mathrm{OP}$ by targeted inhibition of the expression of its target gene Runx2.

In conclusion, the expression level of miR-205 is obviously increased in female patients with T2DM + OP and elderly female mouse model of T2DM + OP. In addition, miR-205 can regulate the osteogenic differentiation of BMSCs, and miR-205/Runx 2 may serve as a new method and target for the treatment of female patients with $\mathrm{T} 2 \mathrm{DM}+\mathrm{OP}$, which provides theoretical support and experimental basis for the research on bone loss in female patients with T2DM + OP.

\section{Acknowledgements}

Not applicable.

\section{Funding}

This study was supported by the Project of Qiqihar Science and Technology (no. SFZD-2017029).

\section{Availability of data and materials}

The datasets used and/or analyzed during the present study are available from the corresponding author on reasonable request.

\section{Authors' contributions}

GZ, HL, WZ, ML, LT and WJ conceived and designed this study. GZ, HL, WZ, ML, WJ and XL conducted the data collection, analysis and summary. GZ, HL, WZ, LT, WJ and $\mathrm{XL}$ were responsible for data analysis and interpretation. GZ wrote the manuscript. All authors read and approved the manuscript and agree to be accountable for all aspects of the research in ensuring that the accuracy or integrity of any part of the work are appropriately investigated and resolved.

\section{Ethics approval and consent to participate}

The study was approved by the Ethics Committee of the Animal Ethics Care Committee of Qiqihar Medical University (QMU-AECC-2019-51).

\section{Patient consent for publication}

Not applicable.

\section{Competing interests}

The authors declare that they have no competing interests.

\section{References}

1. Picke AK, Campbell G, Napoli N, Hofbauer LC and Rauner M: Update on the impact of type 2 diabetes mellitus on bone metabolism and material properties. Endocr Connect 8: R55-R70, 2019.

2. Liu M, Lu Y, Cheng X, Ma L, Miao X, Li N, Sun B, Yan S, Li J and Li C: Relationship between abnormal glucose metabolism and osteoporosis in Han Chinese men over the age of 50 years. Clin Interv Aging 14: 445-451, 2019.

3. Chen FP, Kuo SF, Lin YC, Fan CM and Chen JF: Status of bone strength and factors associated with vertebral fracture in postmenopausal women with type 2 diabetes. Menopause 26: 182-188, 2019.

4. Pagnotti GM, Styner M, Uzer G, Patel VS, Wright LE, Ness KK, Guise TA, Rubin J and Rubin CT: Combating osteoporosis and obesity with exercise: Leveraging cell mechanosensitivity. Nat Rev Endocrinol 15: 339-355, 2019.

5. Schepper JD, Irwin R, Kang J, Dagenais K, Lemon T, Shinouskis A, Parameswaran N and McCabe LR: Probiotics in Gut-bone signaling. Adv Exp Med Biol 1033: 225-247, 2017.

6. Rizzo S, Farlay D, Akhter M, Boskey A, Recker R, Lappe J and Boivin G: Variables reflecting the mineralization of bone tissue from fracturing versus nonfracturing postmenopausal nonosteoporotic Women. JBMR Plus 2: 323-327, 2018.

7. Hadjiargyrou $\mathrm{M}$ and Komatsu DE: The therapeutic potential of microRNAs as orthobiologics for skeletal fractures. J Bone Miner Res 34: 797-809, 2019.

8. Pan BL, Tong ZW, Li SD, Wu L, Liao JL, Yang YX, Li HH, Dai YJ, Li JE and Pan L: Decreased microRNA-182-5p helps alendronate promote osteoblast proliferation and differentiation in osteoporosis via the Rap1/MAPK pathway. Biosci Rep 38: pii: BSR20180696, 2018.

9. Cui Q, Xing J, Yu M, Wang Y, Xu J, Gu Y, Nan X, Ma W, Liu H and Zhao H: Mmu-miR-185 depletion promotes osteogenic differentiation and suppresses bone loss in osteoporosis through the Bgn-mediated BMP/Smad pathway. Cell Death Dis 10: 172, 2019.

10. Li H, Fan J, Fan L, Li T, Yang Y, Xu H, Deng L, Li J, Li T, Weng X, et al: MiRNA-10b reciprocally stimulates osteogenesis and inhibits adipogenesis partly through the TGF- $\beta /$ SMAD2 signaling pathway. Aging Dis 9: 1058-1073, 2018.

11. Li X, Ning L, Zhao X and Wan S: MicroRNA-543 promotes ovariectomy-induced osteoporosis through inhibition of AKT/ p38 MAPK signaling pathway by targeting YAF2. J Cell Biochem: Dec 2, 2018 doi: 10.1002/jcb.28143 (Epub ahead of print).

12. Kim BJ, Lee JY, Park SJ, Lee SH, Kim SJ, Yoo HJ, Rivera De Pena SI, McGee-Lawrence M, Isales CM, Koh JM and Hamrick MW: Elevated ceramides 18:0 and 24:1 with aging are associated with hip fracture risk through increased bone resorption. Aging (Albany NY) 11: 9388-9404, 2019.

13. Sun Y, Xiong Y, Yan C, Chen L, Chen D, Mi B and Liu G: Downregulation of microRNA-16-5p accelerates fracture healing by promoting proliferation and inhibiting apoptosis of osteoblasts in patients with traumatic brain injury. Am J Transl Res 11: 4746-4760, 2019. 
14. Lozano Calderón SA, Garbutt C, Kim J, Lietz CE, Chen YL, Bernstein K, Chebib I, Nielsen GP, Deshpande V, Rubio R, et al: Clinical and molecular analysis of pathologic fracture-associated osteosarcoma: MicroRNA profile Is different and correlates with prognosis. Clin Orthop Relat Res 477: 2114-2126, 2019.

15. Wang Y, Chen $\mathrm{H}$ and Zhang $\mathrm{H}$ : Tanshinone IIA exerts beneficial effects on fracture healing in vitro and in vivo. Chem Biol Interact 310: 108748, 2019.

16. Kaneko T, Okamura K, Yonemoto Y, Okura C, Suto T, Tachibana M, Sakane H, Inoue M and Chikuda H: Effects of denosumab on bone mineral density and bone turnover markers in rheumatoid arthritis patients switching from bisphosphonates. J Exp Orthop 6: 41, 2019.

17. Cehic M, Lerner RG, Achten J, Griffin XL, Prieto-Alhambra D and Costa ML: Prescribing and adherence to bone protection medications following hip fracture in the United Kingdom: Results from the World Hip Trauma Evaluation (WHiTE) cohort study. Bone Joint J 101-B: 1402-1407, 2019.

18. Yang F, Yang L, Li Y, Yan G, Feng C, Liu T, Gong R, Yuan Y, Wang N, Idiiatullina E, et al: Melatonin protects bone marrow mesenchymal stem cells against iron overload-induced aberrant differentiation and senescence. J Pineal Res: 63: e12422, 2017.

19. Lopes D, Martins-Cruz C, Oliveira MB and Mano JF: Bone physiology as inspiration for tissue regenerative therapies. Biomaterials 185: 240-275, 2018.

20. Muruganandan S, Govindarajan R and Sinal CJ: Bone marrow adipose tissue and skeletal health. Curr Osteoporos Rep 16: 434-442, 2018.

21. Fakhry M, Hamade E, Badran B, Buchet R and Magne D: Molecular mechanisms of mesenchymal stem cell differentiation towards osteoblasts. World J Stem Cells 5: 136-148, 2013.

22. Sun MH, Wang WJ, Li Q, Yuan T and Weng WJ: Autologous oxygen release nano bionic scaffold composite miR-106a induced BMSCs enhances osteoblast conversion and promotes bone repair through regulating BMP-2. Eur Rev Med Pharmacol Sci 22: 7148-7155, 2018
23. Luo Y, Zhang Y, Miao G, Zhang Y, Liu Y and Huang Y: Runx1 regulates osteogenic differentiation of BMSCs by inhibiting adipogenesis through Wnt/ $\beta$-catenin pathway. Arch Oral Biol 97: 176-184, 2019.

24. Qing Y, Huang M, Cao Y, Du T and Song K: Effects of miRNA-342-3p in modulating Hedgehog signaling pathway of human umbilical cord mesenchymal stem cells by down-regulating Sufu. Oral Dis 25: 1147-1157, 2019.

25. Yang C, Liu X, Zhao K, Zhu Y, Hu B, Zhou Y, Wang M, Wu Y, Zhang C, Xu J, et al: miRNA-21 promotes osteogenesis via the PTEN/PI3K/Akt/HIF-1 $\alpha$ pathway and enhances bone regeneration in critical size defects. Stem Cell Res Ther 10: 65, 2019.

26. Hu N, Feng C, Jiang Y, Miao Q and Liu H: Regulative effect of Mir-205 on osteogenic differentiation of bone mesenchymal stem cells (BMSCs): Possible role of SATB2/Runx2 and ERK/ MAPK pathway. Int J Mol Sci 16: 10491-10506, 2015.

27. Shi J, Folwaczny M, Wichelhaus A and Baumert U: Differences in RUNX2 and P2RX7 gene expression between mono- and coculture of human periodontal ligament cells and human osteoblasts under compressive force application. Orthod Craniofac Res 22: 168-176, 2019.

28. Yang L, Zeng Z, Kang N, Yang JC, Wei X and Hai Y: Circ-VANGL1 promotes the progression of osteoporosis by absorbing miRNA-217 to regulate RUNX2 expression. Eur Rev Med Pharmacol Sci 23: 949-957, 2019.

29. Polo-Corrales L, Latorre-Esteves M and Ramirez-Vick JE: Scaffold design for bone regeneration. J Nanosci Nanotechnol 14: 15-56, 2014.

This work is licensed under a Creative Commons Attribution-NonCommercial-NoDerivatives 4.0 International (CC BY-NC-ND 4.0) License. 